\title{
FENÓIS HALOGENADOS E/OU SULFATADOS DE MACROALGAS MARINHAS
}

\section{Luciana Retz de Carvalho}

Instituto de Botânica - Secretaria do Meio Ambiente - Av. Miguel Stefano, 3687 - 04301-012 - São Paulo-SP

Nidia F. Roque

Depto de Química Fundamental - Instituto de Química - USP - Av. Lineu Prestes, 748 - 05508-9000 - São Paulo-SP; Departamento de Química Orgânica - Universidade Federal da Bahia - 40177-290 - Salvador - BA

\begin{abstract}
HALOGENATED AND/OR SULFATED PHENOLS FROM MARINE MACROALGAE. During the biological evolution, marine macroalgae have developed biochemicals tools in order to utilize components of seawater such as sulfates and halogens, to produce a variety of chemicals (secondary metabolites).This review shows and discuss the occurrence of sulfated and/or halogenated phenolic compounds in seaweeds.
\end{abstract}

Keywords: seaweed; halogenated phenols; sulfated phenols.

\section{INTRODUÇÃO}

O oceano é resultado de uma gigantesca reação ácido base, onde os ácidos que continuamente afloram do interior da terra são misturados com bases que estão sendo liberadas constantemente das rochas, pela ação do tempo. Suas águas são comparadas a um meio de cultura de composição praticamente constante, o que é verdadeiro para a quase totalidade de seus parâmetros, como por exemplo a porcentagem dos sais dissolvidos, que mantem-se em torno de 3,5\%. Este equilíbrio é sustentado por uma série de reações químicas e de processos físico-químicos ${ }^{1,2}$.

A vida teve origem nos oceanos. Nesse imenso laboratório, os organismos aquáticos e o ambiente abiótico estão interrelacionados e interagem entre si. Por isso, organismos marinhos adaptaram-se às altas concentrações de sais do meio e desenvolveram mecanismos para utilizar os solutos disponíveis ${ }^{3,4,5}$.

Dentre os mais importantes íons presentes no ambiente marinho estão o sulfato, o cloreto, o brometo e o iodeto, em concentrações de $885,19000,65$, e $0,06 \mathrm{mg} . \mathrm{L}^{-1}$, respectivamente $e^{1,2}$. Portanto, não é de surpreender que os organismos marinhos produzam substâncias halogenadas e sulfatadas em abundância, com maior ou menor eficiência.

De todos os organismos marinhos produtores dessas substâncias, sobressaem as algas vermelhas (Rhodophyta), capazes de sintetizar metabólitos halogenados da mais fascinante diversidade, que podem pertencer a praticamente todas as classes químicas, desde hidrocarbonetos de baixo peso molecular, cetonas simples, fenóis, acetogeninas até sofisticados terpenos ${ }^{3}$. Além disso, são eficientes na produção de polissacarídeos sulfatados, os carragenanos e o ágar, que chegam a representar mais de $70 \%$ do seu peso seco e que têm razoável valor comercial ${ }^{6,7,8,9}$.

As algas marinhas pardas (Phaeophyta) e verdes (Chlorophyta) são menos eficientes na produção de substâncias halogenadas, sendo que ambas, e particularmente as pardas, apresentam polissacarídeos sulfatados ${ }^{7,8}$.

As macroalgas marinhas pertencem ao grupo não homogêneo de organismos denominado "Algae", que se encontra subdividido entre os Reinos Monera e Protista. Esses organismos são fotossintetizantes, não vasculares, contêm clorofila a e apresentam estruturas reprodutivas simples, isto é, sem a proteção de células esteréis ${ }^{8,10,11}$.Enquadram-se nesse conceito desde corpúsculos unicelulares microscópicos até os gigantescos "kelps", algas pardas (Phaeophyta) gigantescas que podem alcançar $60 \mathrm{~m}$ de comprimento. Tal variedade de organismos encontra-se distribuída por diferentes "habitats": oceanos, corpos de água doce, solos, rochas e mesmo, superfície de vegetais ${ }^{8,10}$.

As algas foram dispostas em onze Divisões ${ }^{8,10}$, segundo critérios químicos, citológicos e morfológicos, de acordo com os tipos e as combinações de pigmentos fotossensibilizantes presentes, a natureza química das substâncias de reserva e das paredes celulares, a ausência ou a presença de flagelos, o padrão e o curso da mitose e da citocinese, a presença ou a ausência de membrana no retículo endoplasmático e o tipo e a complexidade de ciclo de vida ${ }^{8,10}$.

As macroalgas marinhas vermelhas, pardas e verdes pertencem respectivamente às divisões Rhodophyta, Phaeophyta e Chlorophyta.

\section{EVOLUÇÃO E METABOLISMO DE ALGAS}

Diferentes grupos de algas adaptaram-se ao ambiente marinho através de diferentes vias evolucionárias, determinadas pelas informações genéticas existentes, resultando em diferentes estratégias de sobrevivência, as quais influenciaram enormemente a morfologia, a fisiologia e a bioquímica das macroalgas marinhas, ao longo de seus históricos de vida ${ }^{12,13,14}$. Essa interação com o meio causou diferenças significativas em determinadas características desses organismos, como nas pigmentações. Os pigmentos, além de serem fotossintetizantes (clorofila, carotenóides e ficobiliproteínas), conferem às algas grande poder de adaptação à luz incidente ${ }^{8,15,16,17}$.

Algumas características morfológicas, como a forma geral, o tamanho e a dureza do talo, e determinadas características bioquímicas, como a produção de defesas químicas estão relacionadas com o sucesso das algas nas relações com os herbívoros, de comprovada importância evolucionária e ecológica ${ }^{18,19,20}$.

A dureza do talo é uma das mais importantes defesas físicas contra determinadas espécies de herbívoros ${ }^{21,22}$ enquanto que as defesas químicas são efetivas contra outras ${ }^{19,23}$. Existem casos em que a dureza do talo aparece associada às defesas químicas ${ }^{24}$.

Como defesas químicas, as macroalgas marinhas produzem terpenos, acetogeninas, derivados de aminoácidos, fenóis simples e polifenóis, substâncias que diferem dos produtos de plantas por serem, muito frequentemente, halogenados. Os alcalóides, importantes defesas nas plantas superiores, parecem ser raros ou inexistentes nas macroalgas marinhas, a julgar pela ausência de citações na literatura ${ }^{23,26}$. 
No ambiente marinho, o nitrogênio é considerado fator limitante do crescimento de algas, por se apresentar em baixa concentração ${ }^{2}$, de cerca de $0,5 \mathrm{mg} . \mathrm{L}^{-1}$. Entretanto, sob condições de maior disponibilidade, o nitrogênio pode ser estocado nas rodófitas na forma de nitrato ou como pigmentos fotossintetizantes, ácidos aminados e proteínas ${ }^{27,28}$. Todavia, os alcalóides são encontrados em grupos capazes de fixar $\mathrm{N}_{2}$, como as cianobactérias ${ }^{29,30}$

\section{METABÓLITOS SULFATADOS E/OU HALOGENADOS DE MACROALGAS MARINHAS}

\section{Substâncias sulfatadas}

São numerosas as substâncias sulfatadas isoladas de organismos marinhos ${ }^{31}$ que parecem estar envolvidas na transferência do enxofre inorgânico para o orgânico, pois somente em ambientes com altas concentrações do ânion sulfato são observados organismos capazes de acumulá-lo. A sulfatação é também um modo efetivo de tornar determinadas substâncias solúveis em água, favorecendo, assim, sua excreção. Finalmente, o grupo sulfato poderia ter o papel químico de estabilizador de grupos hidroxílicos de fenóis e de grupos nitrogenados ${ }^{32}$.

A incorporação do sulfato às substâncias orgânicas ocorre por uma reação direta, intermediada por nucleotídeos sulfatados ${ }^{33,34}$.

Nas algas marinhas, os metabólitos primários sulfatados mais importantes e frequientes são os polissacarídeos de Rhodophyta. Esses polímeros formam as famílias de galactanos, como o ágar e o carragenano, de estruturas baseadas na repetição resíduos de galactose e de 3,6-anidrogalactose, unidos por ligações $\beta(1 \varnothing 4)$ e $\alpha(1 \varnothing 3)$, respectivamente ${ }^{6,7,9,10}$. Os galactanos diferem entre si pela quantidade de grupos éster de sulfato e/ou pela presença de outros substituíntes, como grupos metílicos e pirúvicos, formando a matriz flexível que embebe o esqueleto fibroso da parede celular ${ }^{6,7,9,10}$.

Em Phaeophyta, a matriz componente da parede celular é formada pelos fucanos, heteropolissacarídeos sulfatados, cujas cadeias são compostas por fucose, xilose e ácido glucorônico, e em algumas espécies, também por galactose e manose. Nestes polímeros, o grupo sulfato encontra-se ligado ao C-4 da L-fucose e nunca à xilose ou ao ácido glucorônico ${ }^{6,7}$.

Em Chlorophyta, como nas algas vermelhas e pardas, os heteropolissacarídeos sulfatados presentes formam a matriz da parede celular. Estes constituem dois grupos principais, os glucoronoxilorramanos e os arabinoxigalactanos, que formam cadeias altamente ramificadas, os primeiros compostos por ácido glucorônico, xilose e ramnose, estando o grupo sulfato ligado ao C-2 da ramnose, e os últimos, por arabinose, xilose e galactose ${ }^{7}$.

Os polissacarídeos sulfatados devem sua importância econômica ao fato de serem atóxicos e possuírem propriedades gelatinizantes e espessantes, o que lhes atribui considerável valor comercial ${ }^{35,36,37}$. Apresentam também, atividades farmacológicas $^{38,39}$ e são as mais estudadas substâncias sulfatadas de $\operatorname{algas}^{31,32,40}$.

São conhecidos poucos metabólitos secundários sulfatados de algas que pertençam a outras classes químicas: apenas raros glicerídeos $^{41}$, ribosídeos ${ }^{42,43,44}$, lipídeos ${ }^{45}$ e esteróides ${ }^{46}$, além de um expressivo grupo de fenóis. Esses fenóis sulfatados são encontrados nas rodófitas e feófitas. A Tabela 1 apresenta os fenóis sulfatados isolados de macroalgas marinhas.

\section{Substâncias halogenadas}

Dentre as macroalgas, são as rodófitas que apresentam maior aptidão para sintetizar produtos halogenados. O cloro, o bromo e o iodo estão envolvidos nos processos metabólicos dessas algas, sendo o bromo mais freqüentemente incorporado. Por exemplo, espécies de Laurencia, o gênero mais estudado, produzem terpenos bromados, usados, inclusive, como seus marcadores taxonômicos ${ }^{64,65,66}$ e espécies de Polysiphonia biossintetizam fenóis bromados ${ }^{3}$.Os terpenos halogenados de Laurencia e de outros gêneros estão compilados em diversas outras revisões ${ }^{67,68,69}$.

Os fenóis bromados englobam desde fenóis simples $\left(\mathrm{C}_{6}\right)$ a polímeros complexos. Sobre os primeiros existe farta bibliografia $^{70,71}$. O lanosol (éter metílico do álcool 2,3-dibromo-4,5diidroxibenzílico), por exemplo, está entre os bromofenóis mais amplamente distribuídos e é encontrado tanto em micro quanto em macroalgas, sendo bastante documentado na literatu$\mathrm{ra}^{3,70,71,72,73}$. A Tabela 2 apresenta os oligofenóis halogenados produzidos por macroalgas marinhas.

O conhecimento de que halogênios são constituíntes de algas remonta aos anos 20. Durante a $2^{\mathrm{a}}$ Guerra Mundial, o bromo foi extraído, em escala industrial, da rodófita Rhodomela larix, espécie rica em bromofenóis ${ }^{89}$.

Os bromofenóis apresentam bioatividade. O lanosol, por exemplo, é estimulador do crescimento de sementes ${ }^{90}$ e apresenta, também, como outros bromofenóis simples, efeito algicida $^{91}$, antiinflamatório ${ }^{92}$ e antibiótico ${ }^{50}$.

Entre os oligômeros, o vidalol A e o vidalol B apresentam atividade antiinflamatória ${ }^{78}$, o raussonol e o isorraussonol são inibidores da biossíntese do colesterol ${ }^{87,88}$, o colpol é citotóxico $^{81}$ e o avrainvileol tem propriedades antifitofágicas ${ }^{85}$.

Os oligômeros de estrutura baseada no floroglucinol (substâncias 26, 27 e 28 ) formam, com polímeros de mesma origem e de alto peso molecular, os florotaninos, exudados pelas algas pardas para adesão algal ao substrato ${ }^{93}$. Os florotaninos estão contidos em vesículas chamadas fisóides e apresentam diversas atividades biológicas tais como antibacteriana, antialgal, antilarval, antifitofágica. Podem quelar íons de metais pesados ${ }^{94}$, o que torna as algas pardas potenciais indicadores de poluição ${ }^{95}$. Além disso, o material fenólico, após ser liberado pelas algas, vai constituir os compostos húmicos marinhos, dos quais o 2,5-dissulfato de potássio do 1,2,3,5tetraidroxibenzeno (substância 6) é considerado precursor ${ }^{60,61}$,

\section{Biossíntese de substâncias fenólicas}

A origem biossintética da grande maioria das substâncias fenólicas é indicada pelo seu padrão de hidroxilação. Caso a biossíntese tenha ocorrido a partir dos ácidos aminados fenilalanina ou tirosina, os monofenóis resultantes são p-hidroxilados e os polifenóis, o-di ou 1,2,3 triidroxilados. Eles podem assemelhar-se ao catecol, à hidroquinona ou ao pirogalol, dependendo do grau de hidroxilação ${ }^{70,96,97}$

$\mathrm{Na}$ biossíntese via acetato/malonato, os fenóis formam-se pela condensação cabeça-cauda de quatro unidades de acetato, ocorrendo, após a ciclização, reações de descarboxilação, redução e oxidação. As substâncias assim obtidas são metahidroxiladas e dependendo do número de hidroxilas presentes, repetem o padrão do resorcinol ou do floroglucinol ${ }^{70,96,97}$.

A bromação ocorre depois da construção do anel aromático sendo mediada por enzimas bromoperoxidases, das quais se conhecem duas classes: as FeHeme-bromoperoxidases (Fe-BrPO) e as vanádio-bromoperoxidases (V-BrPO), as mais abundantes ${ }^{98}$. As bromoperoxidases catalizam a oxidação do brometo por peróxido de hidrogênio, num processo concomitante com a halogenação de substratos orgânicos adequados ${ }^{98,99,100}$.

A condensação de anéis fenólicos biossintetizados por rotas iguais ou distintas dá origem aos oligofenóis. Entretanto, são poucos os estudos sobre estas condensações ${ }^{74}$.

A análise das Tabelas 1 e 2 evidencia nitidamente a diferença de origem biossintética dos fenóis e oligofenóis pertencentes a rodófitas, clorófitas e feófitas. As duas primeiras biossintetizam as substâncias fenólicas a partir dos ácidos aminados tirosina ou fenilalanina, enquanto que a última o faz através da rota do acetato/malonato ${ }^{70,96,97}$. 
Tabela 1. Substâncias sulfatadas isoladas de macroalgas marinhas.

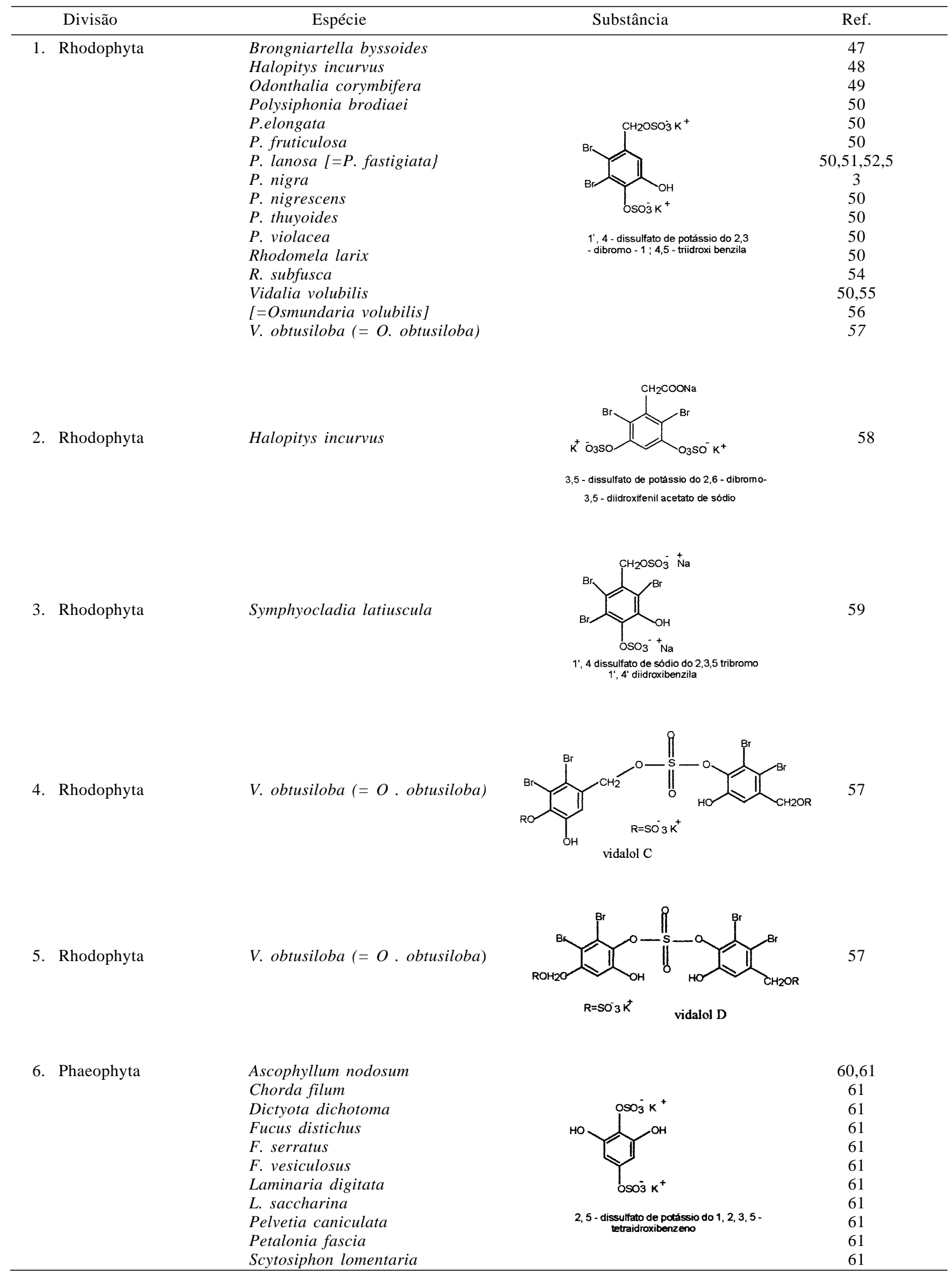


cont. Tabela 1.

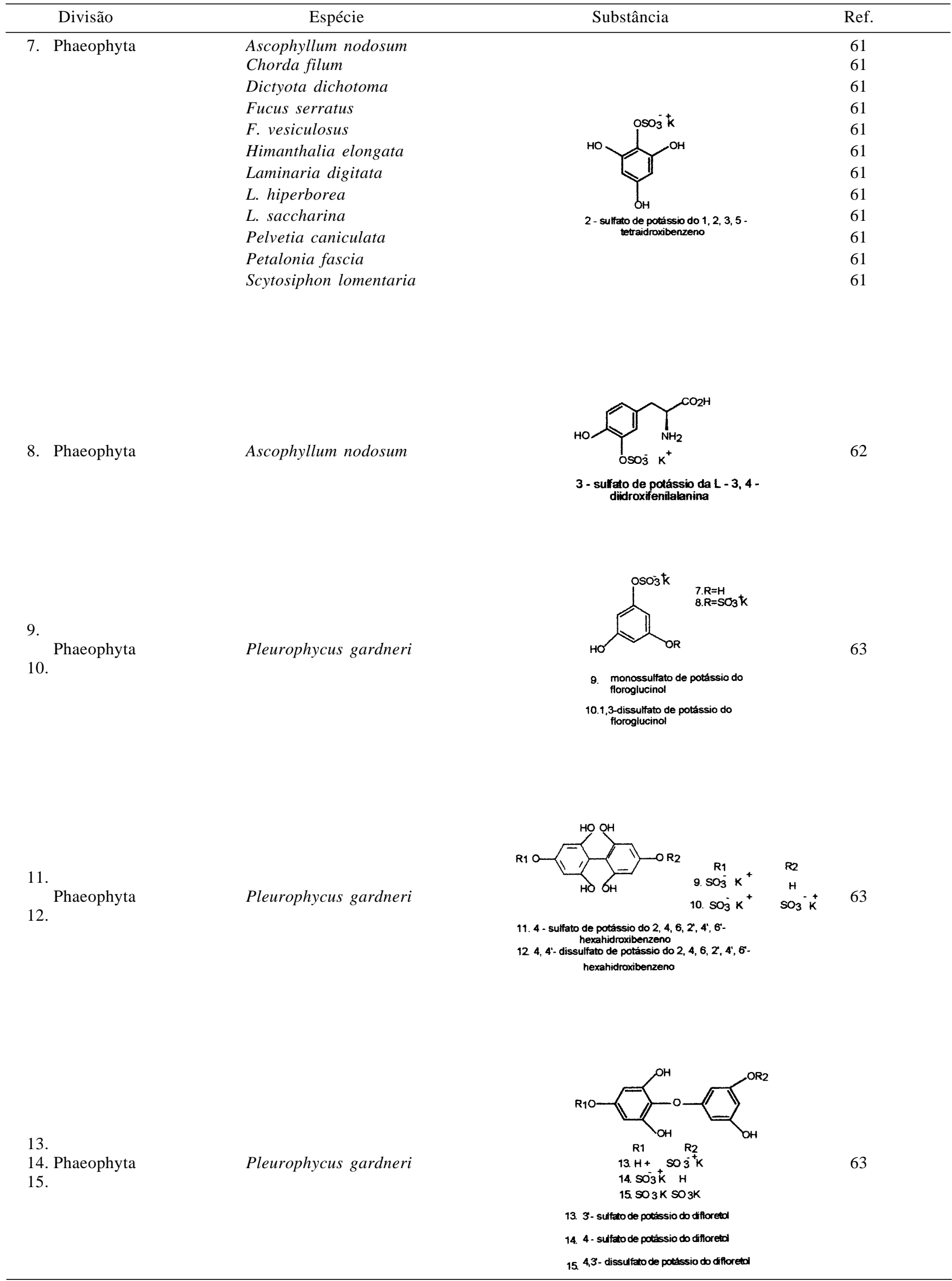


Tabela 2. Oligofenóis de macroalgas marinhas.

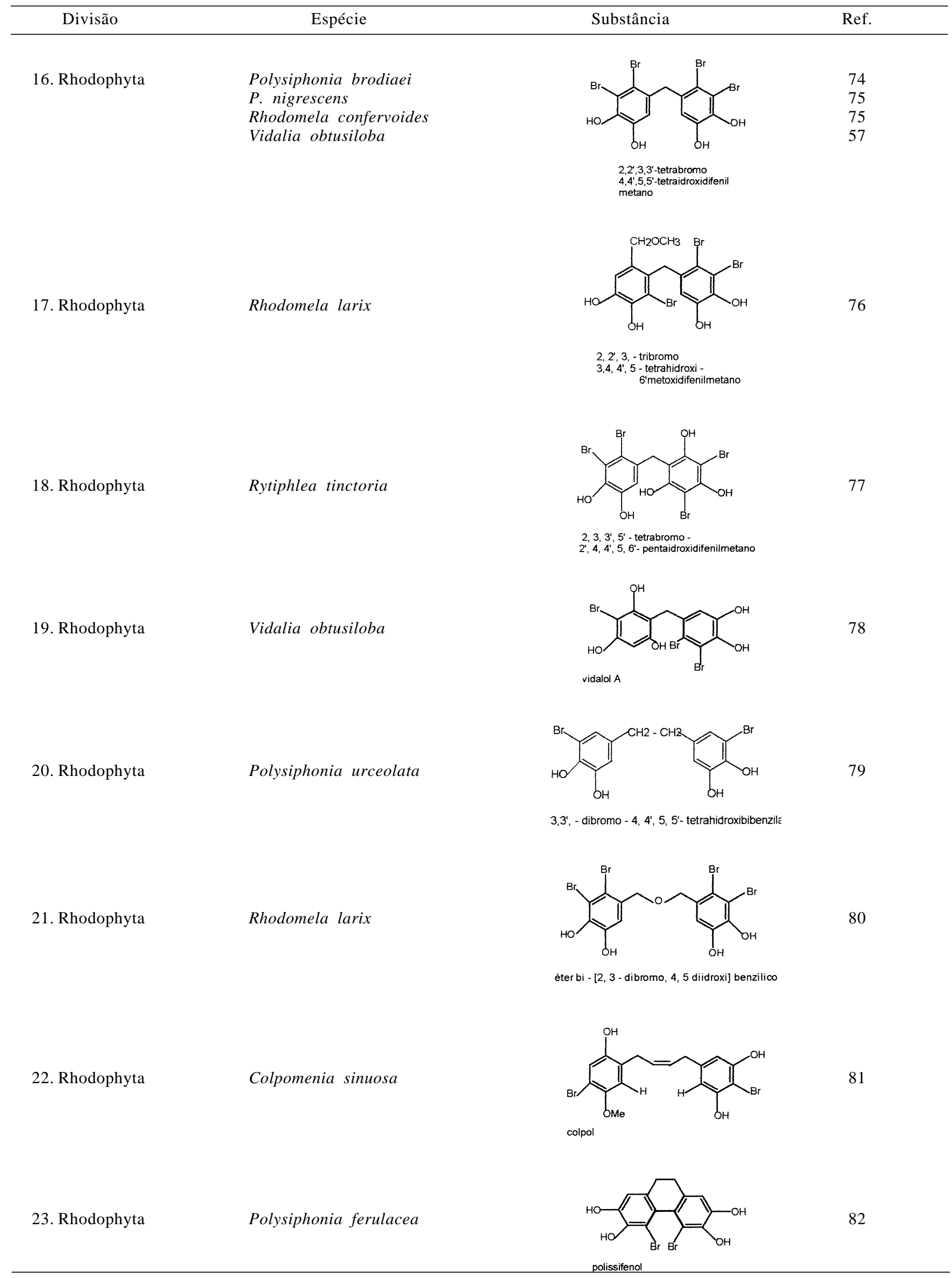


cont. Tabela 2.

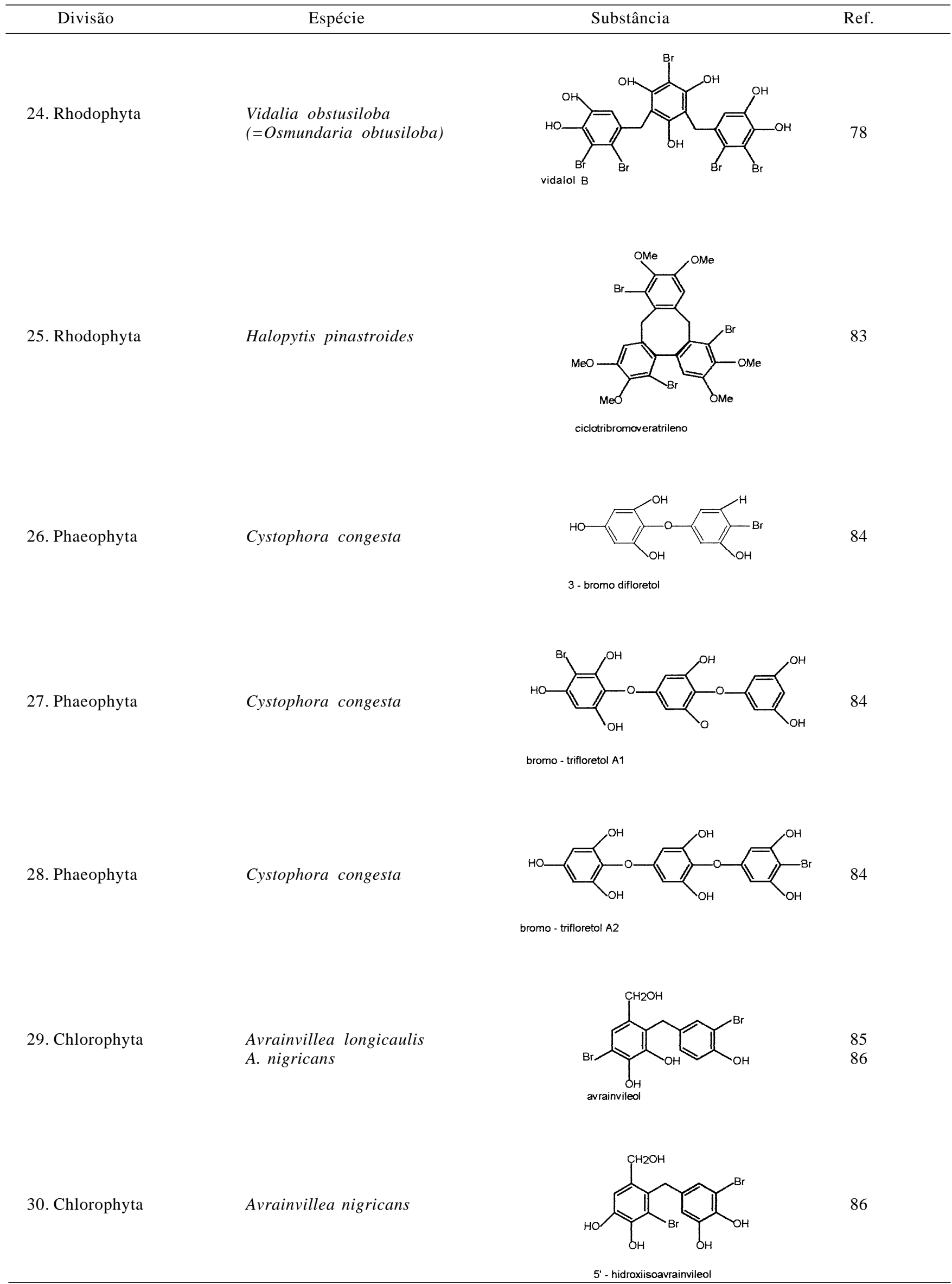


cont. Tabela 2 .

31. Chlorophyta Espécie Avrainvillea rawsonii

\section{REFERÊNCIAS}

1. Stumm, W.; Morgan, J. J.; Aquatic Chemistry - An Introduction Emphasizing Chemical Equilibria in Natural Waters; $2^{\text {nd }}$ Ed.; John Wiley \& Sons, Eds.; Pasadena, 1981.

2. Margalef, R.; Ecologia; Ediciones Ômega S. A.; Barcelona, 1977.

3. Fenical, W.; J. Phycol. 1975, 11, 245.

4. Kanno, N.; Sato, M.; Biochem. Intern. 1990, 21, 519.

5. Kanno, N,; Sato, M.; Sato, Y.; Bot. Mar. 1990, 33, 369.

6. McCandless, E. L.; Craigie, J. S.; Ann. Rev. Plant Physiol. 1979, 30, 41

7. Percival, E.; McDowell, R. H.; in Biochemistry of Storage Carbohydrates in Green Plants; Dey, P.; Dixon, R. A., Eds.; Academic Press; Nova York, 1985, p 305.

8. Hoeck, C. van den; Mann, D. G.; Jahnns, H. M.; Algae. An Introduction to Phycology; Cambridge University Press; Cambridge, 1997.

9. Craigie, J. S.; in Biology of the Red Algae ; Cole, K. M.; Sheath, R. G., Eds.; Cambridge University Press; Cambridge, 1990, p 221.

10. Lee, E.; Phycology; Cambridge University Press; Cambridge, 1989.

11. Ragan, M. A.; Bot. J. Linn. Soc. 1995, 118, 81.

12. Grime, J. P.; Am. Nat. 1977, 111, 1169.

13. Hay, M. E.; Ecology 1981, 62, 739.

14. Littler, M. M.; Littler, D. S.; J. Phycol. 1983, 19, 229.

15. Govindjee; Braun, B. Z.; in Algal Physiology and Biochemistry; Stewart, W. D. P., Ed.; University of California Press; Berkeley, 1974, p 346.

16. Jensen, S. L.; in Marine Natural Products Chemistry; Faulkner, D. J.; Fenical, W. H., Eds.; Plenum Press; New York, 1977, p 239.

17. Glazer, A. N.; West, J. A.; Chan, C.; Biochem. Sist. Ecol. 1982, 10, 203.

18. Padilla, D. K.; Mar. Biol. 1985, 90, 103.

19. Gaines, S. D.; Lubchenco, J.; Ann. Rev. Ecol. Syst. 1982, $13,111$.

20. Hay, M. E.; Oecologia 1984, 64, 396.

21. Steinberg, P. D.; Ecol. Mon. 1985, 55, 333.

22. Schupp, P. J.; Paul, V. J.; Ecology 1994, 75, 1172.

23. Hay, M. E.; Fenical, W.; Ann. Rev. Ecol. Syst. 1988, 19, 111.

24. Hay, M. E. ; Kappel, Q. E.; Ecology 1994, 75, 1714.
25. Cronin, G.; Hay, M. E.; Ecology 1996, 77, 2287.

26. a) Faulkner, D. J.; Tetrahedron 1977, 11, 1421;

b) Faulkner,D. J.; Nat. Prod. Rep. 1984, 1, 251;

c) Faulkner, D. J.; Nat. Prod. Rep. 1986, 3, 6;

d) Faulkner, D. J.; Nat. Prod. Rep. 1987, 4, 539;

e) Faulkner, D. J.; Nat. Prod. Rep. 1990, 7, 269;

f) Faulkner, D. J.; Nat. Prod. Rep. 1991, 8, 103;

g) Faulkner, D. J.; Nat. Prod. Rep. 1992, 9, 323;

h) Faulkner, D. J.; Nat. Prod. Rep. 1993, 10, 497;

i) Faulkner, D. J.; Nat. Prod.Rep. 1994, 11, 355;

j) Faulkner, D. J.; Nat. Prod. Rep. 1995, 12, 223;

k) Faukner, D. J.; Nat. Prod. Rep. 1996, 13, 75;

1) Faulkner, D. J.; Nat. Prod. Rep. 1998, 15, 259.

27. Naldi, M.; Wheeler, P. A.; J. Phycol. 1999, 35, 70.

28. Lapointe, B. E.; J. Phycol. 1981, 17, 90.

29. Sivonem, K.; Jones, G.; in Toxic Cyanobacteria in Water - A Guide to Their Public Health Consequences, Monitoring and Management; Chorus, I; Bartram, J. Eds.; E \& FN Spon; Londres, 1999, p 399.

30. Harada, K.; in Toxic Microcystis; Watanabe, M. F.; Harada, K.; Carmichael, W. W.; Fujiki, H. Eds.; CRC Press; Boca Raton, 1996, p 262.

31. Kornprobst, J. N.; Sallenave, C.; Barnathan, G.; Comp. Biochem. Physiol. 1998, 119 B, 1.

32. Kanno, N.; Sato, M.; Biochem. Intern. 1990, $21,519$.

33. Rennenberg, H.; Ann. Rev. Plant Physiol. 1984, 35, 121.

34. Kanno, N.; Sato, M.; Sato, Y.; Bot. Mar. 1990, 33, 369.

35. McLachlam, J.; Plant and Soil 1985, 89, 137.

36. Oliveira, E. C.; Panorama da Aqüicultura 1997, maio/junho, 24.

37. Oliveira, E. C.; Panorama da Aqüicultura 1997, julho/ agosto, 42.

38. Neushul, M.; Hydrobiologia 1990, 204/205, 99.

39. Franz, G.; Planta Med. 1989, 55, 493.

40. Usov, A I.; Foods Hydrocolloids 1992, 6, 9.

41. Liem, P. Q.; Laur, M.H.;Biochemie 1976, 58, 1367.

42. Edmonds, J. S.; Morita, M.; Shibata, W.; J. Chem. Soc.. Perkin Trans I 1987, 577.

43. Francesconi, K. H.; Edmonds, J. S.; Stick, R. V.; Skelton, B. W.; White, A. H.; J. Chem. Soc. Perkin Trans. I 1991, 2707. 44. Shibata, Y.; Morita, M.; Agric. Biol. Chem. 1998, 52, 1087. 45. Liem, P. Q.; Laur, M. H.; Biochemie 1976, 58, 1381.

46. Govindam, M.; Abbas, S. A.; Schmitz, F. J.; Lee, R. H.; Papkoff, J. S.; Slate, D. L.; J. Nat. Prod. 1994, 57, 74. 
47. Fries, L.; Experientia 1973, 29, 1436.

48. Angier, J.; Mastagli, P.; Comptes rendus 1956, 242, 190.

49. Kurata, K.; Amiya, T.; Yabe, K.; Bull. Jap. Soc. Sci. Fish.; 1973, 39, 973.

50. Glombitza, K. W.; Stoffelen, H.; Murawsky, U.; Bielaczec, J.; Egge, H.; Planta Med. 1974, 25, 107.

51. Angier, J.; Hoffmann, G.; Bull. Soc. Bot. Fr. 1952, 99, 80.

52. Hodgkin, J. H.; Craigie, J. S.; McInnes, A. G.; Can. J. Chem. 1966, 44, 74.

53. Glombitza, K. W.; Stoffelen, H.; Planta Med. 1972, 22, 391.

54. Weinstein, B.; Rold, T. L.; Harrel, C. E.; Burns, M. W.; Waaland, J. R.; Phytochemistry 1975, 14, 2667.

55. Kurata, K.; Amiya, T.; Jap. Soc. Sci. Fish. 1975, 41, 657.

56. Angier, J.; Henry, M. H.; Bull. Soc. Bot. Fr. 1950, 97,29.

57. Carvalho, L. R.; Tese de Doutorado; Instituto de Química - Universidade de São Paulo, SP, 1999.

58. Nanteuil, G.; Mastagli, P.; Phytochemistry 1981, 20, 1750.

59. Kurata, K.; Amiya, T.; Chem. Lett. 1980, 279.

60. Jensen, A.; Ragan, M. A.; Tetrahedron Lett. 1978, 9, 847.

61. Ragan, M. A.; Jensen, A.; Phytochemistry 1979, 18, 261.

62. Laycock, M. V.; Ragan, M. A.; J. Nat. Prod. 1984, 47, 1033.

63. Glombitza, K. W.; Knoss, W.; Phytochemistry 1992, 31, 279.

64. Fenical, W.; Norris, J. N.; J. Phycol. 1975, 11, 101.

65. Masuda, M.; Abe, T.; Suzuki, T.; Suzuki, M.; Phycologia 1996, 35, 550 .

66. Masuda, M.; Abe, T.; Sato, S.; J. Phycol. 1997, 33, 196.

67. Erickson, K. L.; in Marine Natural Products: Chemical and Biological Perspectives; Scheuer, P. J., Ed.; vol.V, chap. 4, Academic Press; New York, 1983; p 131.

68. Accorinti, J.; Algas-Fuente de Nuevos Fármacos; Instituto Antártico Argentino; Buenos Aires, 1988.

69. Pereira, R. C.; Teixeira, V. L.; Quim. Nova 1999, 22, 369.

70. Higa, T.; in Marine Natural Products. Chemical and Biological Perspectives; Scheuer, P. J.,Ed,;vol. IV. chap. 3; Academic Press; New York, 1983; p 93.

71. Gribble, G, W.; in Progress in the Chemistry of Organic Natural Products ; Herz, W.; Grisebach, H.; Kirby, G. W., Eds.; vol.LXVIII; Springer; New York, 1983; p 1.

72. Pedersen, M.; Da Silva, E. J.; Planta 1973, 115, 83.

73. Pedersen, M.; Fries, L.; F. Pflanzenphysiol 1975, 74, 272.

74. Lundgren, L.; Olsson, K.; Theander, O.; Acta Chem. Scand. 1979, B 33, 105.
75. Pedersen, M.; Phytochemistry 1978, 291.

76. Kurata, K.; Amiya, T.; Chem. Lett. 1977, 1435.

77. Chevolot-Magueur, A. A.;Cave, A.; Riche, C; Phytochemistry 1976, 15, 767.

78. Wiemer, D. F.; Idler, D. D.; Fenical, W.; Experientia 1991, 47, 851 .

79. Kurata, K., Amiya, T.; Nakano, N.; Chem. Lett. 1976, 821.

80. Suzuki, M.; Kowata, N., Kurosawa, E.; Bull. Chem. Soc. Jpn. 1980, 53, 2099.

81. Green, D.; Kashmann, Y.; J. Nat. Prod. 1993, 56, 1201.

82. Aknin, M.; Samb, A.; Mirailles, J.; Costantino, V.; Fattorusso, E.; Mangoni, A.; Tetrahedron Lett. 1992, 33, 555.

83. Combaut, G.; Chantraine, J. N.; Teste, J.; Glombitza, K. W.; Phytochemistry 1978, 17, 1791.

84. Kock, M.; Gregson, R. P.; Phytochemistry 1984, 23, 2633.

85. Sun, H.H.; Paul, V.J.; Fenical, W.; Phytochemistry 1983, 22, 743.

86. Colon, M.; Guevara, P.; Gerwick, W. H.; J. Nat. Prod. 1987, 28, 2917.

87. Chen,J. L.; Gerwick, W. H.; J. Nat. Prod. 1994, 57, 947.

88. Carte, B. K.; Troupe, N.; Chan, J. A.; Westley, J. W.; Faukner, J.; Phytochemistry 1989, 28, 2917.

89. Craigie, J. S.; Gruenig, D. E.; Science 1967, 157, 1058.

90. Kubo, I.; Ochi, M.; Shibata, K.; Hanke, F. J.; Nakatsu, T.; Tan, K. S.; J. Nat. Prod. 1990, 53, 50.

91. McLachlan, J.; Craigie, J. S.; J. Phycol 1966, 2, 133.

92. Niang, L. L.; Hung, X.; Hydrobiologia 1984, 116, 168.

93. Vreeland, V.; J. Phycol. 1998, 34, 1.

94. Ragan, M. A.; Can. J. Chem. 1985, 63, 294.

95. Bryan, G. W.; Hummerstone, L. G.; J. Mar. Biol. Ass. U.K. 1973, 53, 705.

96. Neish, A. C.; in Biochemistry of phenolic compounds; Harbone, J. B.,Ed.; Academic Press; New York, 1964; p 295.

97. Mann, J.; Secondary Metabolism; $2^{\text {nd }}$ ed.; Atkins, P. W.; Holker, J. S. E.; Holliday, A. K.; Eds.; Oxford University Press; Oxford, 1996.

98. Buttler, A.; Walker, J. V.; Chem. Rev. 1993, 93, 1937.

99. Everett, R. R.; Kanofsky, J. R.; Buttler, A.; J. Biol. Chem. 1990, 265, 4908.

100. Krenn, B. E.; Plat, H.; Wever. R.; Biochim. Biophys. Acta 1987, 912, 28. 